\section{Radiographic basal ganglia abnormalities secondary to nonketotic hyperglycemia with unusual clinical features}

\author{
Ju Young Choi, Joon Min Park, Kyung Hwan Kim, Jun Seok Park, \\ Dong Wun Shin, Hoon Kim, Woo Chan Jeon, Hyun Jong Kim
}

Department of Emergency Medicine, Inje University Ilsan Paik Hospital, Inje University College of Medicine, Goyang, Korea

A 77-year-old woman was admitted to a local clinic for altered consciousness and presented with a suspected basal ganglion hemorrhage detected on brain computed tomography. The patient was stuporous, but her vital signs were stable. Her initial blood glucose was $607 \mathrm{mg} / \mathrm{dL}$, and a hyperdense lesion was found in the right basal ganglion on brain computed tomography. T1-weighted magnetic resonance imaging revealed high signal intensity in the right basal ganglion. Electroencephalography showed no seizure activity. The patient was treated with a fluid infusion, and serum glucose level was controlled with insulin. The patient gradually recovered consciousness and was alert within 24 hours as serum glucose level normalized. The basal ganglion lesion caused by hyperglycemia was not accompanied by involuntary limb movement. This is the first report of a patient presenting with decreased consciousness and typical neural radiographic changes associated with nonketotic hyperglycemia but without movement abnormalities.

Keywords Hyperglycemia; Basal ganglia; Tomography, X-ray computed; Magnetic resonance imaging

eISSN: 2383-4625

Received: 8 August 2016

Revised: 26 August 2016

Accepted: 27 August 2016

Correspondence to: Joon Min Park Department of Emergency Medicine, Inje University Ilsan Paik Hospital, Inje University College of Medicine, 170 Juhwa-ro, Ilsanseo-gu, Goyang 411-706, Korea E-mail: aero7@hanmail.net

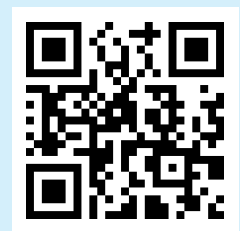

\section{Capsule}

Summary

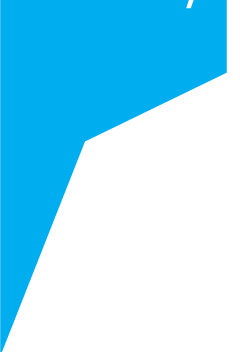

What is already known

Hyperdense basal ganglion lesions on brain computed tomography have been reported in nonketotic hyperglycemic patients mainly in association with involuntary limb movement.

\section{What is new in the current study}

The same radiographic abnormality was found in a nonketotic hyperglycemic patient with altered consciousness only. Clinicians should consider nonketotic hyperglycemia even in the absence of a movement disorder.
How to cite this article:

Choi JY, Park JM, Kim KH, Park JS, Shin DW, Kim H, Jeon WC, Kim HJ. Radiographic basal ganglia abnormalities secondary to nonketotic hyperglycemia with unusual clinical features. Clin Exp Emerg Med 2016;3(4):252-255.

This is an Open Access article distributed under the terms of the Creative Commons Attribution Non-Commercial License (http:// creativecommons.org/licenses/by-nc/3.0/). 


\section{INTRODUCTION}

Brain computed tomography (CT) is commonly used to assess intracranial abnormalities in the emergency department. Possibilities that should be considered in the differential diagnosis of a hyperdense basal ganglion lesion on brain CT include benign calcification, hypertensive intracerebral hemorrhage (ICH), presence of a foreign body, Tay-Sachs disease, and metabolic diseases such as hyperglycemia. ${ }^{1}$ The basal ganglion is a common anatomic site for hypertensive $\mathrm{ICH}$; thus, emergency physicians are familiar with these CT findings. Several studies have reported patients with nonketotic hyperglycemia whose CT findings mimic those of patients with ICH. These patients mainly present with a contralateral movement disorder referred to as diabetic hemichorea-hemiballism (HC-HB). ${ }^{1-4}$ Patients with diabetic HC-HB usually present with involuntary, continuous unilateral movement abnormalities in both the proximal and distal extremities. ${ }^{5}$ However, not all patients with hyperglycemia and a hyperdense basal ganglion lesion present with an abnormal movement disorder. For instance, Hansford et al. ${ }^{6}$ reported a patient with these imaging abnormalities without a movement disorder. Similarly, the present report details a patient with nonketotic hyperglycemia and a hyperdense basal ganglion lesion with an unusual clinical presentation.

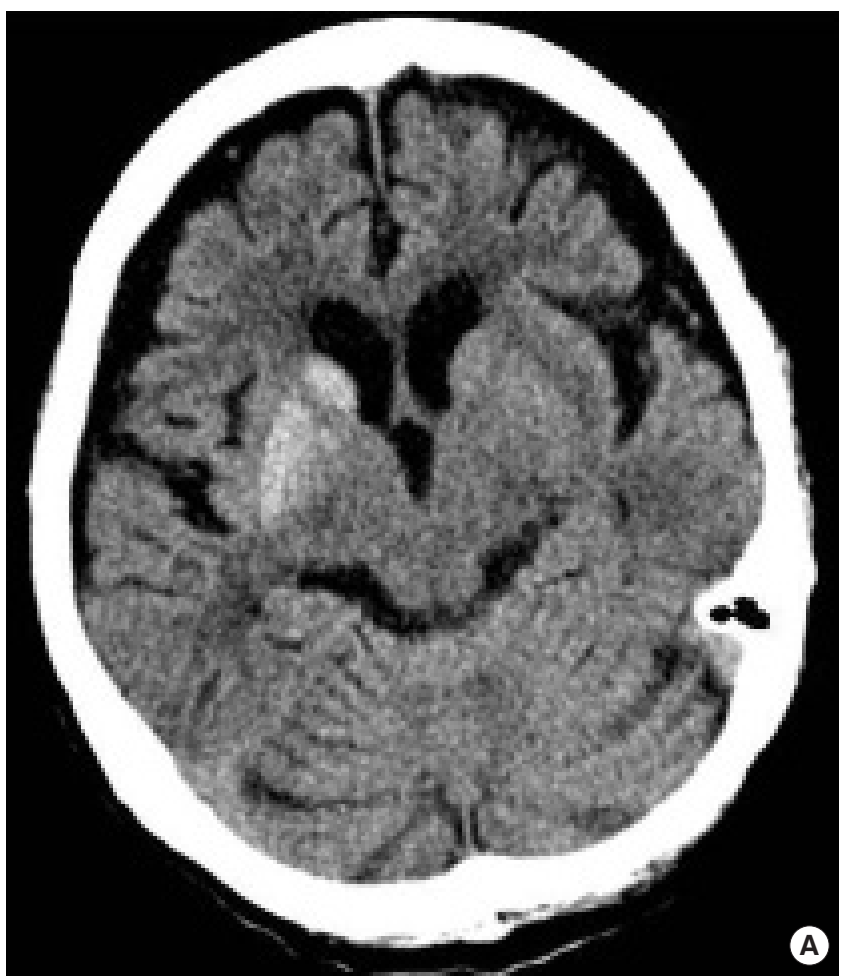

\section{CASE REPORT}

A 77-year-old woman lost consciousness 6 hours before being admitting to a local clinic where she underwent brain CT. The patient was promptly referred to our department with suspected basal ganglion ICH based on the brain CT. The patient had no medical history other than diabetes. Her premorbid Eastern Cooperative Oncology Group score was 3 due to dementia but she had normal cognitive functioning. The patient had stopped taking her diabetic medication 2 months earlier, on her own volition, and had been suffering progressive debilitation. No other symptoms were present. The patient did not take any sedatives or tranquilizers. The initial vital signs were blood pressure $180 / 98 \mathrm{mmHg}$, pulse rate $110 / \mathrm{min}$, respiratory rate $18 / \mathrm{min}$, and body temperature $36.8^{\circ} \mathrm{C}$. A neurological examination revealed stupor, intact brainstem signs, and greater than grade III motor strength in all four extremities without lateralizing signs. She did not exhibit any abnormal movements of the extremities. No specific abnormalities were found on a systemic physical examination. The initial serum laboratory test results were blood glucose $607 \mathrm{mg} / \mathrm{dL}$, hemoglobin $12.8 \mathrm{~g} / \mathrm{dL}$, white blood cells $6,720 / \mathrm{mm}^{3}$, platelets $193,000 / \mathrm{mm}^{3}$, aspartate aminotransferase $32 \mathrm{IU} / \mathrm{L}$, alanine aminotransferase $38 \mathrm{IU} / \mathrm{L}$, blood urea nitrogen $17 \mathrm{mg} / \mathrm{dL}$, serum cre-

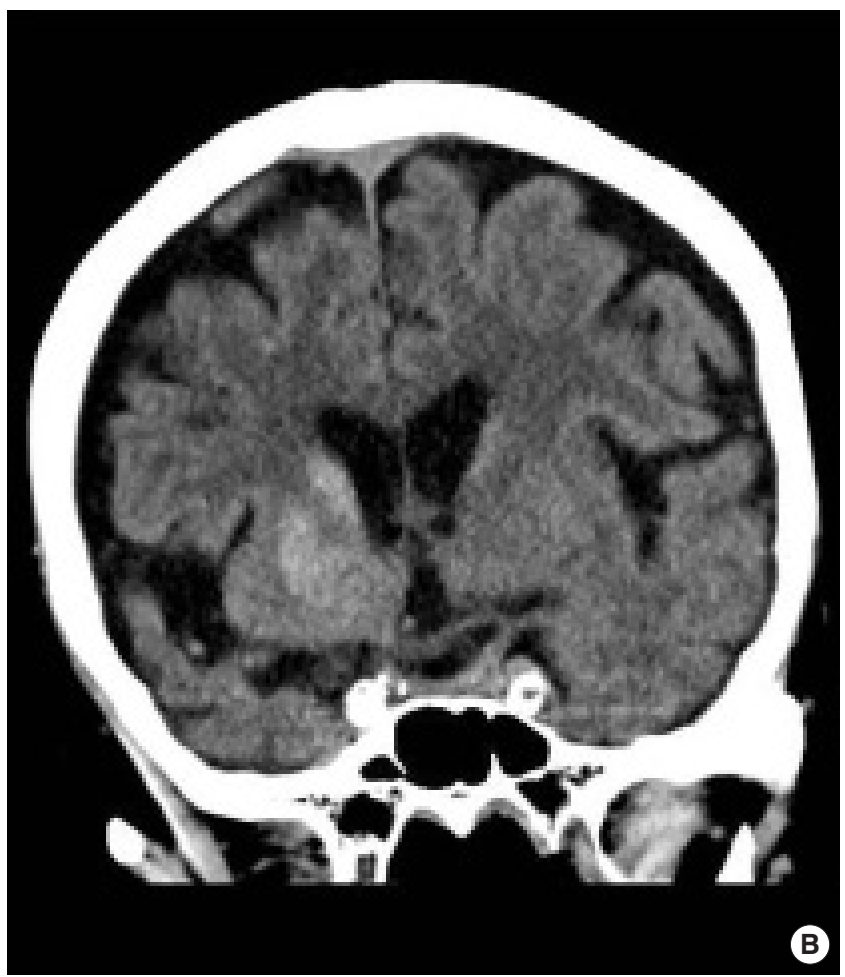

Fig. 1. Axial (A) and coronal (B) brain computed tomography. The right basal ganglion shows a hyperdense lesion. The internal capsule had normal density. No mass effect was observed. 

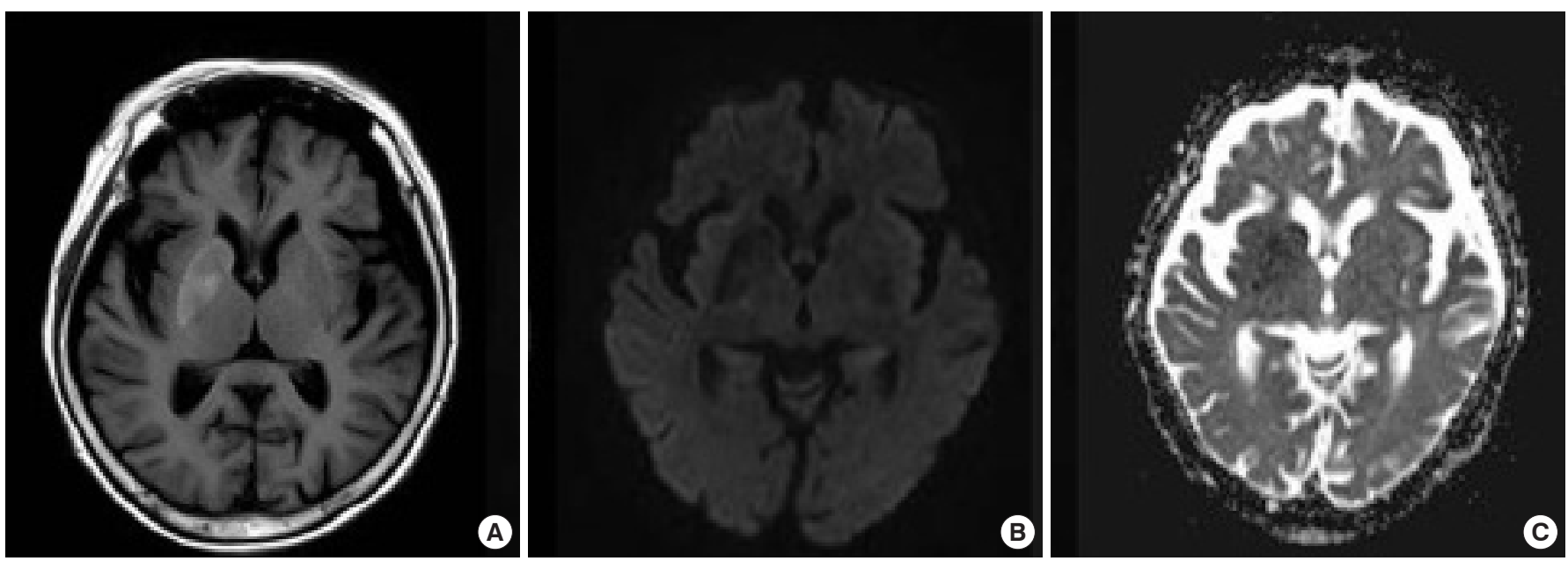

Fig. 2. Brain magnetic resonance imaging shows high signal intensity on a T1-weighted image (A), low signal intensity on a diffusion-weighted image (B), and low signal intensity on the apparent diffusion coefficient map (C). No mass effect was observed.

atinine $1.2 \mathrm{mg} / \mathrm{dL}$, sodium $120 \mathrm{mEq} / \mathrm{L}$, potassium $5.0 \mathrm{mEq} / \mathrm{L}$, chloride $89 \mathrm{mEq} / \mathrm{L}$, serum osmolarity $280 \mathrm{m0sm}$, and lactate $12.5 \mathrm{mg} /$ $\mathrm{dL}$. No serum ethanol was detected, and an arterial blood gas analysis revealed the following: $\mathrm{pH} 7.454, \mathrm{pCO}_{2} 37.0 \mathrm{mmHg}, \mathrm{pO}_{2}$ $78.1 \mathrm{mmHg}, \mathrm{HCO}_{3} 25.4 \mathrm{mmol} / \mathrm{L}$, and base excess $1.6 \mathrm{mmol} / \mathrm{L}$. No ketones were detected in the urinalysis. Barbiturates, benzodiazepines, and tricyclic antidepressants were not found during a toxin-screening test. The initial brain CT showed a hyperdense lesion in the right basal ganglion (Fig. 1). On magnetic resonance imaging, T1-weighted images revealed high signal intensity indicative of changes due to nonketotic hyperglycemia; diffusion weighted imaging (DWI) and an apparent diffusion coefficient map showed restricted diffusion (Fig. 2). Blood glucose level was controlled with regular insulin, and conservative treatment and fluid resuscitation were provided. Electroencephalography revealed findings compatible with diffuse encephalopathy, but no signs of seizure were detected. After admission to the intensive care unit, the patient gradually recovered consciousness and was alert after 24 hours, as blood glucose and sodium were controlled. No abnormal movement disorder was noted after a return to consciousness. The patient was discharged 10 days after admission without any complications.

\section{DISCUSSION}

Diabetic HC-HB is a rare complication of nonketotic hyperglycemia in which patients usually present with continuous unilateral involuntary movement. This condition is more prevalent in elderly East Asian women with poorly controlled diabetes. ${ }^{7}$ Most patients have typical radiographic basal ganglia abnormalities contralateral to the affected side. ${ }^{8}$ The predominant CT findings are hyper- attenuation of the putamen with or without hyperattenuation of the caudate nucleus, sparing the internal capsule. Magnetic resonance imaging findings include high signal intensity on T1-weighted images, variable signal intensity on T2-weighted images, and restricted diffusion on DWI. ${ }^{18-10}$ Emergency physicians can misdiagnose this condition as acute ICH if not familiar with these radiographic abnormalities, considering the acute nature of the clinical symptoms and similarities on $\mathrm{CT}$ findings. Contrary to diabetic $\mathrm{HC}-\mathrm{HB}$, acute basal ganglion hemorrhage usually involves peripheral edema and cerebral parenchymal displacement due to mass effect. $^{6}$

Several hypotheses regarding the pathogenesis of radiographic abnormalities have been proposed. Petechial hemorrhage was initially considered as the main mechanism underlying hyperdensity on CT and high signal intensity on T1-weighted images. ${ }^{9}$ However, such radiographic abnormalities typically adhere to neuroanatomical boundaries rather than vascular territories. ${ }^{6}$ Another study found no hemosiderin deposits in an anatomical structure with abnormal radiographical findings." An alternative hypothesis is that hyperviscosity induced by hyperglycemia and vasogenic edema play a role in radiological abnormalities as they induce restricted diffusion on DWI and apparent diffusion coefficient maps. ${ }^{12}$ Similarly, hyperviscosity may cause partial ischemic injury to the striatum, which results in high signal intensity on T1-weighted images. ${ }^{11}$ Hsu et al. ${ }^{13}$ proposed that metabolic derangements related to hyperglycemia and cerebral vascular insufficiency constitute the main underlying mechanism. They found that fluorodeoxyglucose uptake in the basal ganglion decreased based on results from a positron emission tomography analysis. Another possible hypothesis is that excess formation of gemistocytes, which are reactive swollen forms of astrocytes, result from ischemic chan- 
ges and metabolic derangement. In line with this theory, Shan et al. ${ }^{4}$ found several gemistocytes in a stereotactic putamen biopsy specimen, claiming that these changes led to high signal intensity on T1-weighted images. Hence, while various hypotheses have been suggested, the main mechanisms underlying radiographic abnormalities remain unclear.

With the exception of two documented patients, all patients with nonketotic hyperglycemia and radiographic abnormalities have also presented with an involuntary movement disorder. Specifically, Hansford et al..$^{6}$ reported a patient who complained of vague stroke-like symptoms, and Hsu et al. ${ }^{13}$ reported a patient who had unilateral limb weakness, gait disturbance, and speech difficulties. Similar to these two cases, the present report observed the same radiographic abnormalities without a movement disorder, but the clinical presentation only included diminished consciousness. Although altered consciousness in a patient with hyperglycemia can be caused by increased serum osmolarity, electrolyte imbalance, or prolonged metabolic acidosis, ${ }^{14}$ such results did not apply to the present patient. We concluded that the patient developed a form of metabolic encephalopathy, considering the electroencephalography results and gradual improvement in consciousness in accordance with corrected blood glucose and sodium. The importance of this case is that radiographic abnormalities observed in a patient with diabetic HC-HB can also be observed in patients presenting only with altered consciousness.

Although radiographic changes caused by nonketotic hyperglycemia are uncommon, emergency physicians should consider typical radiographic features. In addition, emergency physicians should remember that clinical features likely involve various signs and symptoms apart from a movement disorder.

\section{CONFLICT OF INTEREST}

No potential conflict of interest relevant to this article was reported.

\section{REFERENCES}

1. Wilson TJ, Than KD, Stetler WR Jr, Heth JA. Non-ketotic hyperglycemic chorea-hemiballismus mimicking basal ganglia hemorrhage. J Clin Neurosci 2011;18:1560-1.

2. Verma R, Praharaj HN. Hemichorea-hemiballism as the pre- senting manifestation of diabetes mellitus. BMJ Case Rep 2013; 2013.

3. Branca D, Gervasio O, Le Piane E, Russo C, Aguglia U. Chorea induced by non-ketotic hyperglycaemia: a case report. Neurol Sci 2005;26:275-7.

4. Shan DE, Ho DM, Chang C, Pan HC, Teng MM. Hemichoreahemiballism: an explanation for MR signal changes. AJNR Am J Neuroradiol 1998;19:863-70.

5. Wang JH, Wu T, Deng BQ, Zhang YW, Zhang P, Wang ZK. Hemichorea-hemiballismus associated with nonketotic hyperglycemia: a possible role of inflammation. J Neurol Sci 2009;284: 198-202.

6. Hansford BG, Albert D, Yang E. Classic neuroimaging findings of nonketotic hyperglycemia on computed tomography and magnetic resonance imaging with absence of typical movement disorder symptoms (hemichorea-hemiballism). J Radiol Case Rep 2013;7:1-9.

7. Bordelon YM, Smith M. Movement disorders in pregnancy. Semin Neurol 2007;27:467-75.

8. Oh SH, Lee KY, Im JH, Lee MS. Chorea associated with nonketotic hyperglycemia and hyperintensity basal ganglia lesion on T1-weighted brain MRI study: a meta-analysis of 53 cases including four present cases. J Neurol Sci 2002;200:57-62.

9. Lai PH, Tien RD, Chang MH, et al. Chorea-ballismus with nonketotic hyperglycemia in primary diabetes mellitus. AJNR Am J Neuroradiol 1996;17:1057-64.

10. Zaitout Z. CT and MRI findings in the basal ganglia in non-ketotic hyperglycaemia associated hemichorea and hemi-ballismus (HC-HB). Neuroradiology 2012;54:1119-20.

11. Ohara S, Nakagawa S, Tabata K, Hashimoto T. Hemiballism with hyperglycemia and striatal T1-MRI hyperintensity: an autopsy report. Mov Disord 2001;16:521-5.

12. Chu K, Kang DW, Kim DE, Park SH, Roh JK. Diffusion-weighted and gradient echo magnetic resonance findings of hemichorea-hemiballismus associated with diabetic hyperglycemia: a hyperviscosity syndrome? Arch Neurol 2002;59:448-52.

13. Hsu JL, Wang HC, Hsu WC. Hyperglycemia-induced unilateral basal ganglion lesions with and without hemichorea. A PET study. J Neurol 2004;251:1486-90.

14. Scott $A$, Claydon A, Brennan $G$. The management of the hyperosmolar hyperglycaemic state (HHS) in adults with diabetes. London: Diabetes UK; 2012. 